\title{
The impact of dietary supplement NT-020 with Rhodiola rosea on energy, fatigue, and perceived exertion
}

\section{Priscila Lamadrid ${ }^{1}$, Danielle K. Williams ${ }^{1}$, Marcus W. Kilpatrick ${ }^{1}$, Paula C. Bickford ${ }^{3}$, and Cyndy D. Sanberg ${ }^{2}$}

${ }^{1}$ College of Education ${ }^{1}$, University of South Florida, Tampa, Florida, USA; ${ }^{3}$ Muma College of Business, University of South Florida, Tampa, Florida, USA; ${ }^{3}$ Morsani College of Medicine, University of South Florida, Tampa, Florida, USA

Corresponding Author: Marcus Kilpatrick, PhD, Exercise Science Program, University of South Florida, Tampa, Florida, USA

Submission Date: August $23^{\text {rd }}$, 2019, Acceptance Date: November $30^{\text {th }}$, 2019. Publication Date: November $30^{\text {th }}, 2019$

Citation: Lamadrid P, Williams DK, Kilpatrick MW, Bickford PC, Sanberg CD: The Impact of Dietary Supplement NT-020 with Rhodiola rosea on Energy, Fatigue, and Perceived Exertion. Functional Foods in Health and Disease 2019; 9(11): 706 -718. DOI: https:/doi.org/10.31989/ffhd.v9i11.656

\begin{abstract}
Purpose: Evaluate the effectiveness of a commercially available nutraceutical product on levels of exercise-related energy, fatigue, and exertion before and after 4 weeks of supplementation. Primary ingredients within the product include a proprietary blend of blueberry extract, green tea extract, L-carnosine, Vitamin D3 (NT-020; 920mg), and Rhodiola rosea, a plant that purports to boost energy and hold antioxidant and anti-inflammatory properties.
\end{abstract}

Methods: Twenty-seven participants (13 females, 14 males, mean BMI $=23$, mean age $\pm \mathrm{SD}=$ $24 \pm 6$ years) completed a baseline assessment of aerobic fitness (mean VO2 peak $=40 \mathrm{~mL} \mathrm{x} \mathrm{kg}^{-1}$ $\mathrm{x} \mathrm{min}^{-1}$ ) before being randomized into a placebo or supplement group for four weeks. All participants were involved in regular physical activity three or more days per week. Assessment of energy, fatigue, and perceived exertion responses during and immediately following moderately intense cycle ergometry exercise was conducted before and after the 4-week ingestion period during which participants were instructed to maintain existing exercise activities.

Results: Data were analyzed by way of repeated measures ANOVA and dependent t-tests to determine the presence of significant differences across time and between the supplement and placebo conditions. Participants receiving the supplement reported: greater levels of energy and lower levels of fatigue during the initial moments after completing the exercise trial $(\mathrm{p}<0.05)$, greater levels of energy at the midpoint of the exercise trial $(\mathrm{p}<0.05)$ but not at the end of the exercise session $(\mathrm{p}>0.05)$, and lower perceived exertion at four of the six measurement points 
during exercise $(\mathrm{p}<0.05)$. No differences were observed from pre to post intervention within the placebo condition $(\mathrm{p}>0.05)$.

Conclusions: Findings indicate that a commercially available supplement marketed to boost energy and reduce fatigue can deliver the purported benefits at least in part. Related findings that supplementation for a 4-week period can allow for equal work at a lower rating of perceived exertion provides further, though limited support that this product may have efficacy.

Keywords: fatigue; perceived exertion; energy; Rhodiola rosea; NT-020

\section{BACKGROUND}

The use and consumption of products that aim to enhance energy and vitality is a big industry. Recent estimates indicate that the vitamin and dietary supplement industry totals more than \$36 billion annually in the United States alone [1]. An increase in energy and vitality has been seen with the widely-spread use of caffeine. Research indicates that $90 \%$ of Americans use caffeine daily in the form of coffee, soft drinks, or other over-the-counter supplement products in order to increase energy throughout the day [2]. Importantly, research generally supports the widespread belief that caffeine is safe, increases energy, enhances alertness, and benefits exercise performance [3].

A related, but separate category of supplements that has become popular is adaptogens. Adaptogens are labeled as substances that purport to help the body adapt to stress and resist fatigue [4]. Each adaptogen works in different ways to produce its effects.

Among the most commonly utilized adaptogens is Rhodiola rosea, a medicinal plant also known as roseroot or arctic root. It is composed of Rosine Rosarines, Rosin, Tyrosol, Rosiridin, Tannins, Polyphenols, Salidroside, and Rosavin. This root plant product has traditionally been used to increase physical endurance, treat fatigue, treat stress and anxiety, prevent altitude sickness, and assist in stimulating the nervous system [5]. Rhodiola rosea naturally activates the production of norepinephrine, serotonin, dopamine, and acetylcholine, which all contribute to the benefits of the natural components of the root [5]. A systematic review noted that 4 weeks of supplementation with Rhodiola rosea accompanied by aerobic exercise reduced lactate levels and muscle damage biomarkers in trained athletes [5]. Another recent systematic review of this agent noted several studies supporting its purported benefits, but also noted the need for more rigorous scientific designs to determine true efficacy [6]. Importantly, side effects associated with the intake of Rhodiola rosea are primarily limited to feeling anxious, jittery, and agitated such that it is recommended that consumption in the hours before sleep be avoided [7].

Although there is lack of definitive evidence of the benefits of Rhodiola rosea, the use of this herb is widespread, both as a standalone product and in combination with other nutraceuticals. A particular product utilizing Rhodiola rosea is NT-020 plus Rhodiola rosea, which contains Rhodiola rosea alongside a blend of blueberry extract, green tea extract, L-carnosine, and Vitamin D3 known as NT-020. The primary purported benefit of NT-020 is a synergistic effect on stem cell proliferation, which is vital to help the body to remain healthy and ready to repair as it ages [8]. A limited body of research suggests these purported benefits do occur in response to utilization of NT-020 $[8,9,10]$. There is evidence that each of the individual components has some effect on 
stem cell proliferation, which is the reason they were studied initially. However, NT-020 has been shown to be more than additive to promote stem cell proliferation when the individual ingredients are combined together [8].

The many beneficial properties of blueberry extract, such as its anti-inflammatory properties, are thought to be attributable to their group of bioactive compounds. These bioactive compounds belong to the proanthocyanidin and anthocyanin classes [11]. Another component of NT-020 is green tea extract, which is composed of catechins (most abundant is epigallocatechin gallate or EGCG), theanine, and caffeine. The anti-inflammatory and antioxidant properties of green tea are speculated to come from the EGCG [12]. L-carnosine is composed of beta-alanine and L-histidine, which comprises a dipeptide. It is synthesized in the skeletal muscles and the brain and reported to be involved in cardiovascular function, metabolism, and energy expenditure. It serves a role as an antioxidant, $\mathrm{pH}$ buffer, metalion chelate, and a carbonylation agent. It is seen to be released during physical exercise and exhibits its benefits in a dose-response manner. It can affect blood glucose, body temperature, blood pressure, and lipolysis [13]. Vitamin D is another component of NT-020. It is an essential fat-soluble vitamin and can be synthesized from sunlight exposure and its 2 primary forms are D2 (ergocalciferol) and D3 (cholecalciferol). Both of these differ in their structures, but have similar ways of general metabolism and in functions in the body. Its primary role as a hormone is to assist in regulating calcium homeostasis along with parathyroid hormone (beneficial for bone growth and development). The secondary role is its regulation of immune function and protein synthesis [14].

Other investigations of NT-020 have considered the potential benefits to cognition [15]. The basis of these investigations is the potential to change the environment associated with aging. Importantly, supplementation with NT-020 in animals has been shown to positively impact cellular vitality and induce neurogenesis with associated improvements in cognition [16]. Studies on NT020 have shown positive effects to reduce inflammation, increase Nrf2 mediated oxidative stress response, and increase WNT related stem cell molecular signally [16, 17]. Separately, a study in aging adults noted that NT-020 improved cognitive function by increasing processing speed [15]. These findings could potentially warrant further research into the effects of increased processing speed on measures such as exertion and performance.

Research related to Rhodiola rosea and NT-020 is encouraging but there are still some gaps in our knowledge. While there is reason to believe that the combination of these agents might provide benefit to exercise-related performance through aspects of physical and mental fatigue, research has not yet evaluated this possibility and such an investigation seems appropriate given that the combination is commercially available. Therefore, the purpose of the current study is to evaluate the effectiveness of a commercially available nutraceutical product on levels of energy, fatigue, and exertion before and after 4 weeks of supplementation.

\section{METHODS}

\section{Participants}

Participants were 27 adults (13 females, 14 males, mean age \pm SD $=24 \pm 6$ years) at a large university in the southeastern United States and were recruited by way of electronic mail and word of mouth. All participants were healthy and physically active, defined as participating in at least three days per week of moderate-intensity physical activity [17]. In order to participate in the present study, participants must have met these inclusion criteria: between 18-45 years old and 
designated as low risk for cardiovascular disease based on American College of Sports Medicine Guidelines [16]. Participants were excluded from the study if they met any of the following criteria: moderate or severe obesity (BMI > 35), hypertension (systolic blood pressure of 140 or higher; diastolic blood pressure 90 or higher), current smoker, designated as having a moderate or high risk based on known or suspected cardiovascular, pulmonary, or metabolic disease as determined by standardized health risk status questionnaire or physical examination. The sample size is a reflection of related research and is based on an anticipated medium-to-large effect size (i.e., ES = 0.5 to 0.8 ), a power level of 0.8 , and an alpha criterion of 0.05 . All procedures were approved by the university institutional review board and the research was registered in the appropriate federal database.

\section{Design Overview}

The study utilized a double blind, placebo control design in which participants were randomly assigned to either placebo or supplement conditions. Participants as well as researchers were unaware of supplement assignment until after data analysis. Twenty-seven out of the 30 participants enrolled completed a familiarization day, a maximal exercise test, and two experimental exercise trials separated by four weeks, whereas three participants failed to complete all assessments. Participants dropping out of the study did so for reasons related to time, interest, and availability. The first visit included completion of informed consent procedures and a physical exam. The second visit included the measurement of aerobic capacity by way of a maximal exercise test on a cycle ergometer. The third and fourth visits consisted of 30-minute continuous, steady state cycle ergometer exercise trials.

\section{Screening}

The first meeting in the lab was the familiarization day, where the informed consent was conducted. Participants who were consuming supplements to enhance physical performance were excluded from the study as well as those that planned on starting a new exercise regimen during the four-week span. Those who could not commit to study guidelines were excluded from the study. Participants also completed a medical screen, a physical examination, a health history questionnaire, and baseline data, namely height, weight, resting heart rate, and resting blood pressure. The physical examination was conducted by a licensed medical professional.

\section{Metabolic Testing}

A progressive, ramp protocol was performed on an electronically-braked cycle ergometer (Lode, Groningen, Netherlands). The protocol ramp rate varied between 15-25 watts per minute and was based on a standardized formula [18]. The test was terminated when the participant could not maintain a pedal cadence of $30 \mathrm{rpm}$. Heart rate (HR), blood pressure (BP), ratings of perceived exertion (RPE), and expired gas were monitored in accordance with standard exercise testing guidelines [16]. HR was measured using a HR monitor (Polar, Lake Success, NY) and BP was determined by auscultation. RPE was estimated each minute using the CR-10 Scale [19]. Expired gases were collected through an air cushion mask and analyzed continuously using a metabolic cart (Vacumetrics, Ventura, CA). Peak oxygen consumption $\left(\mathrm{VO}_{2}\right.$ peak), which is the gold standard criterion for aerobic fitness and capacity, was identified as the largest volume of oxygen consumed per minute during the test. Maximal exertion was verified through the observation of 
peak HR (90+\% of age-predicted maximum heart rate), RPE (9 or above on the 0-10 scale), and respiratory exchange ratio (greater than 1.10). The test was terminated volitionally by the participant at the point of fatigue.

\section{Experimental Trials}

The remaining two trials consisted of identical cycle ergometer exercise sessions. Participants were instructed to: avoid strenuous exercise for 24 hours prior, arrive to the lab rested (7-9 hours of sleep) and hydrated, avoid caffeine and energy drinks for 24 hours prior, and to not consume a full meal within 3 hours of research appointments, though a snack prior to testing was allowed if desired. Upon arrival to the lab, participants were instructed to sit and relax, followed by completion of perceptual measures and assessment of HR and BP. Participants then completed a 30-minute continuous cycle ergometer protocol with 2-minute warm-up and cool-down periods for a trial lasting 34 minutes total. The 30 -minute continuous portion was set at a submaximal workload of $50-60 \%$ of the maximal wattage achieved during their maximal exercise test previously conducted. Twenty participants completed their exercise sessions at $60 \%$ peak work capacity and seven participants completed their session at $50 \%$ peak work capacity based on reported difficulty with the $60 \%$ workload. For all exercise trials, research staff was instructed to limit communication with participants to matters related to data collection during the exercise to establish consistency for all trials. Identical laboratory visits occurred for the trials completed at the study's start and completion separated by 28 days with efforts made to complete pre and post testing at similar times during the day.

\section{Experimental Instructions}

Upon completion of the first experimental trial, participants were given an unlabeled bottle containing either the supplement or the placebo and were instructed and reminded, by way of text messaging, to take two capsules (920mg) (which is consistent with label instructions) each morning 1-2 hours upon waking. Participants were given a form to track their exercise during the intervention period and were instructed to write down any physical activity they completed each day. They were told to maintain current exercise regimens without the addition of any new routines for the duration of the intervention period.

\section{Instrumentation}

The primary variables of interest for this study were psychophysiological, namely energy, fatigue, and exertion. Heart rate served as a secondary variable of interest. These variables were assessed before, during, and after exercise at both baseline (prior to initiating supplement or placebo) and follow-up (after 28 days of supplement or placebo). Measurements taken as a baseline upon arrival to the lab before and after the intervention to assess the impact of the supplementation regimen on general energy and fatigue levels utilized a subscale of the Profile of Mood States [21], with five items for the fatigue subscale and six items for the vigor (i.e., energy) subscale. Both subscales asked "How are you feeling right now?" and utilized a 5-point scale ranging from 1 (not at all) to 5 (extremely).

Measurements taken during, and/or after exercise were: rate of perceived exertion (RPE), fatigue, energy, and heart rate (HR). HR and RPE were assessed every five minutes during 
exercise. Fatigue and energy were assessed twice during exercise at 15 and 30 minutes and once after exercise at 1 minute after cool-down. RPE was measured using a validated scale [19] while fatigue and energy were assessed using measures developed for this study utilizing the same scaling as used with RPE. Each of these scales utilized an 11-point scale ranging from 0 (none at all) to 10 (maximal possible). HR was measured using a monitor (Polar, Lake Success, NY).

\section{Statistical Analysis}

Analyses of the data proceeded in three phases. The first phase included descriptive analysis of participant characteristics. The second phase included separate analyses for both time within trial and from pre to post intervention by way of analysis of variance (ANOVA) for heart rate, exertion, energy, and fatigue. Specifically, each dependent variable was analyzed using a 2 (Condition: Supplement, Placebo) x 2 (Time: Pre, Post) for each time point. These analyses allowed for inspection of main effects and interaction effects for each factor. The third phase included individual planned contrasts by way of t-tests to compare means within and across trials. Statistical significance was set at an alpha criterion of 0.05 and effect size (ES) differences were calculated from marginal means to report practical significance between means [22]. Version 24 of SPSS was utilized to analyze data.

\section{RESULTS}

\section{Participant Characteristics}

The participants were normal to marginally overweight (mean BMI $\pm \mathrm{SD}=25 \pm 3 \mathrm{~kg} / \mathrm{m}^{2}$ for both males and females). Maximal exercise testing indicated that the participants were moderately fit for their age (mean $\mathrm{VO}_{2}$ peak $\pm \mathrm{SD}=45 \pm 6 \mathrm{ml} \mathrm{x} \mathrm{kg}^{-1} \mathrm{~min}^{-1}$ for males and $35 \pm 8 \mathrm{ml} / \mathrm{kg} / \mathrm{min}$ for females, mean age $\pm \mathrm{SD}=24 \pm 6$ years) (see Table 1). Testing data revealed the participants had a mean maximal test HR of $188 \pm 7$ beat $\mathrm{min}^{-1}$, a mean maximal RPE of $9.5 \pm 0.8$, and a mean respiratory exchange ratio of $1.20 \pm 0.10$. These data suggest that maximal effort was achieved. Furthermore, results indicate that $93 \%$ of participants $(n=25)$ reached the criterion for perceived effort (RPE $\geq 9), 89 \%$ of participants $(n=24)$ reached the criterion for maximal heart rate (HR $\geq$ $90 \%$ age-predicted maximum), and $93 \%$ of participants $(n=25)$ reached the criterion for maximal respiratory exchange ratio of $\geq 1.10$.

Table 1: Descriptive statistics for the study sample.

\begin{tabular}{|c|c|c|c|}
\hline & Supplement & Placebo & Total \\
\hline Sex & $\begin{array}{l}7 \text { females, } \\
7 \text { males }\end{array}$ & $\begin{array}{l}6 \text { females, } \\
7 \text { males }\end{array}$ & $\begin{array}{l}13 \text { females, } \\
14 \text { males }\end{array}$ \\
\hline Age (years) & $23.4(6.0)$ & $26.0(6.7)$ & $24.6(6.4)$ \\
\hline Body Mass Index & $24(2.9)$ & $25.8(3.2)$ & $24.9(3.1)$ \\
\hline Metabolic Capacity $\left(\mathrm{ml} \mathrm{x} \mathrm{kg}{ }^{-1} \mathrm{~min}^{-1}\right)$ & $39.4(8.6)$ & $40.4(7.6)$ & $39.9(8.0)$ \\
\hline Work Capacity (watts) & $215(49)$ & $241(57)$ & $228(54)$ \\
\hline
\end{tabular}

Note: mean (standard deviation); no significant group differences $(\mathrm{p}>0.05)$. 
Energy and Fatigue Before and After Intervention

Responses for fatigue and vigor showed that there were no significant differences between groups $(p>0.05)$ at baseline or after intervention for either variable, suggesting no impact on general energy and fatigue (see Table 2).

Table 2: Perceptual responses before and after 28-day intervention.

\begin{tabular}{|c|c|c|c|c|}
\hline \multirow[t]{2}{*}{ Time (min) } & \multicolumn{2}{|l|}{ Supplement } & \multicolumn{2}{|l|}{ Placebo } \\
\hline & Day 1 & Day 28 & Day 1 & Day 28 \\
\hline Fatigue & $8.4(3.2)$ & $9.3(3.5)$ & $9.4(4.2)$ & $8.0(2.2)$ \\
\hline Vigor & $16.6(5.8)$ & $18.2(4.2)$ & $18.2(4.9)$ & $18.5(4.0)$ \\
\hline
\end{tabular}

Note: no significant differences for condition, time, or condition $\mathrm{x}$ time interaction $(\mathrm{p}>0.05)$.

\section{Heart Rate and Exertion Responses Relative to Exercise Performance}

A significant effect on heart rate (HR) was observed with HR increasing from the beginning to end of exercise in all trials $(\mathrm{p}<0.05)$. HR responses from pre to post intervention were mostly similar at each respective time point except for an increase in HR at the 5-minute and 10-minute marks of the exercise trial for the supplement group when comparing pre to post intervention, where HR was elevated significantly in the post-intervention exercise trial ( $<<0.05$; ES < 0.5). All other HR values measured pre and post intervention were not significantly different $(\mathrm{p}>0.05)$ (see Table 3).

A significant effect on rating of perceived exertion (RPE) was observed with RPE increasing from the beginning to end of exercise in all trials $(\mathrm{p}<0.05)$. RPE responses from pre to post intervention exhibited numerous differences within the supplement condition but not within the placebo condition. Specifically, while RPE was not different at the 5-minute mark within the supplement condition ( $\mathrm{p}>0.05)$, RPE was lower during the post-intervention trial than the preintervention trial at the 10-, 15-, 20-, 25-, and 30-minute marks ( $\mathrm{p}<0.05$; ES range $=0.4$ to 1.0), suggesting less exertion was required to complete an identical exercise trial after the supplementation period. No differences were observed from pre intervention to post intervention within the placebo condition $(\mathrm{p}>0.05)$ (see Table 3$)$.

\section{Fatigue and Energy Responses Relative to Exercise Performance}

Significant effects on energy and fatigue were observed for specific points of comparison. Specifically, energy was lower during exercise at the pre intervention time point for the supplement condition ( $\mathrm{p}<0.05 ; \mathrm{ES}=0.4$ ), and fatigue was increased during exercise at the pre-intervention time point for both conditions $(\mathrm{p}<0.05$; $\mathrm{ES}=0.5$ ). In contrast, no significant changes were observed at the post-intervention time point for either condition $(\mathrm{p}>0.05)$. One other change was significantly decreased fatigue at the post-intervention time point for the supplement condition ( $p$ $<0.05$; ES $=0.8$ ). No significant changes were observed within the placebo condition $(\mathrm{p}>0.05)$. 
Table 3: Cardiovascular and Perceptual responses.

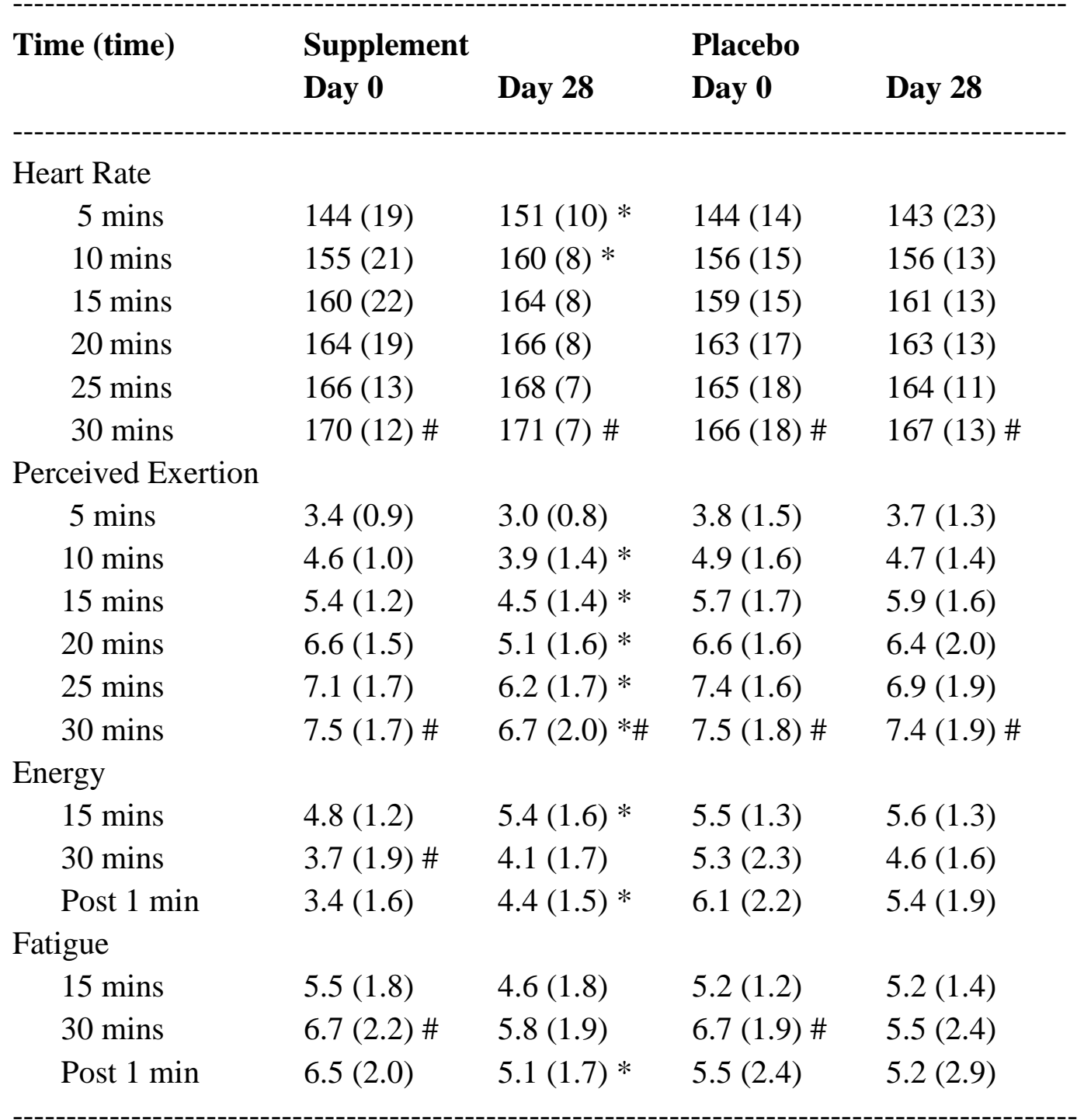

Note: * significant difference from baseline and 28 days $(\mathrm{p}<0.05)$. \# significant difference from first to last measurement during exercise $(\mathrm{p}<0.05)$.

\section{DISCUSSION}

The present experiment was designed to investigate the effects of the daily ingestion of a Rhodiola rosea based supplement product on levels of energy, fatigue, and exertion before, during, and after exercise prior to and after supplementation. A double-blind placebo control design was utilized to address the research questions. The experimental approach required participants to conduct a maximal exercise test followed by submaximal exercise sessions on a cycle ergometer at a workload of approximately $60 \%$ of peak work capacity. Supplementation over a 28 -day period initiated immediately after the baseline exercise trial continued until the day of the final exercise trial. The design yielded a session of exercise that would be typical of healthy, active individuals undertaking recommended aerobic training. The present findings offer mixed results related to the efficacy of this proprietary blend of purported ergogenic ingredients. 
Findings related to cardiovascular responses are secondary compared to primary dependent variables, but suggest that heart rate was largely unaffected by supplementation. As expected, heart rate increased from beginning to end for both the baseline and follow-up exercise sessions, for both the supplement and placebo conditions. This upward drift of heart rate is typical and expected for moderately intense exercise of significant duration [22]. The only differences in heart rate observed from baseline to follow-up was an increase in heart rate for the first 10 minutes within the supplement condition, suggesting that the supplement created a greater cardiovascular stimulus early in exercise that dissipated during the middle and concluding portions of the trial. This outcome was not predicted and cannot be readily accounted for, but what is noteworthy is that the heart rate increase was only modest both in terms of actual heart rate increase (less than 10 beats per minute) and the size of the effect (less than one half standard deviation on average). The overall assessment of the cardiovascular responses before and after supplementation confirm that the supplement ingredients are not cardiovascular stimulants. Thus, any of the purported benefits would arise from other mechanisms. However, Rhodiola rosea has been observed in a recent review to have preventative functions to prevent stress-induced cardiac damage. These effects are seen to have an antiarrhythmic effect, prevent reduced coronary flow, and increase contractility in the post-ischemic period [23].

Findings related to energy and fatigue represent the core outcomes of the study and provide a mixed view of the efficacy of NT-020 with Rhodiola rosea as an ergogenic aid. The mixed findings are reflected by evidence of positive impacts during exercise at follow-up and lack of evidence prior to exercise at follow-up. More specifically, measures of fatigue and energy suggest that 28 days of supplementation did not induce significant changes in a rested state. Participants did not report subjectively feeling more energy or vigor upon entering the lab after four weeks of supplementation, nor did they report less fatigue. These specific findings are not supportive of the purported benefits of the product and suggest either no benefit is available or that the supplementation regimen was insufficient either in duration or dosing. In contrast, findings during and immediately after exercise were more positive. Feelings of energy during exercise were greater in the supplement condition at follow-up when compared to the placebo condition, despite no differences observed during the pre-exercise condition. At follow-up participants reported having significantly more energy during the middle of the exercise session and immediately after exercise ended, though perceptions of energy near the end of the exercise session only trended toward and did not reach significance. Somewhat similar results were observed for measures of fatigue, where participants in the supplement group reported less fatigue immediately after exercise and demonstrated non-significant trends towards significance for fatigue during exercise. These mixed results are consistent with a systematic review of randomized controlled trials that evaluated the impacts of Rhodiola rosea on physical and mental fatigue [6], which noted improvements in physical fatigue in about one third of the surveyed literature and more than half of the studies that focused on mental fatigue. The authors recommended a rigorously-designed and well reported research trial to provide better insights on the efficacy of Rhodiola rosea and the current design provides an incremental step towards developing a full understanding, especially as it relates to subjectively reported energy and fatigue. However, another study has demonstrated that the subjects were able to finish the endurance time trial at a faster pace, but without a significant 
change in mood state [24]. These findings align with our findings and is a step in the appropriate direction for research on this type of supplement.

Among the findings most supportive of Rhodiola rosea combined with blueberry extract, green tea extract, vitamin D3, and carnosine as a beneficial supplement is its link to ratings of perceived exertion during exercise. Exertion ratings were taken every five minutes. These findings provide a consistent pattern whereby participation in the exercise session after the conclusion of the supplementation period was marked by reduced effort at all but one of the six measurement time points. Though there was only a trend towards significance at the 5-minute mark (that included a difference of about one half of a standard deviation), beginning at the 10-minute mark and continuing through the full 30 minutes, it was observed that moderately intense aerobic exercise was perceived as easier for participants at follow-up who were taking the supplement. The observed differences were both significant and also included rather robust effect size differences that ranged from almost two thirds up to a full standard deviation, suggesting both a statistical and practical significance. More specifically, ratings of perceived exertion were reduced after supplementation on average by a full unit on the 0-10 scale and overall represented a reduction from baseline that was no less than $10 \%$ and up to $20 \%$. These changes may be meaningful for active, healthy individuals suggesting that they were able to perform the same amount of work at a reduced effort cost. This could allow for work to be increased compared to pre-supplementation without an increase in perceived exertion.

Strengths and weaknesses of the current design are noteworthy. Primary strengths of the design relate to the utilization of double blinding and a placebo control condition. Such designs reduce bias and address concerns previously brought forth regarding existing research conducted on Rhodiola rosea [6]. Other notable strengths include the sample, which was relatively robust in terms of size and is representative of a wide segment of the general population, and the exercise manipulation that was used, which is reflective of existing recommendations for exercise [18]. Potential weaknesses of the present study include the presence of only two measurement points and the relative short duration of the supplement intervention. Research has not yet elucidated how soon any beneficial impacts may appear or the duration that is necessary to achieve full effects of supplementation, if any.

In addition to Rhodiola rosea, other ingredients in the NT-020 formulation may also have contributed to the effects observed in this study. For example, the green tea extract contains caffeine and a high catechin content. A recent study demonstrates an increase in some aspects of muscle energy substrate utilization [25]. Another study demonstrated that green tea extract increased swimming duration in rats via an interaction with calcium balance regulation in fast twitch muscles [26]. Thus, it is possible that the green tea contributed to the observed effects.

Given the innovations and limitations within the current study, there are opportunities to improve and expand this research line for future studies. While the dosing utilized in the present study followed guidelines provided by the manufacturer, future studies might consider comparing lower and higher dosages over a longer time. Measurement of energy and fatigue could be conducted more frequently before and after intervention to reduce the impacts of individual days that might create outliers and negative impacts on data quality. Future designs might also consider manipulation of the exercise intensity or duration. Based on the significant benefits related to perceived exertion, it could prove valuable to prescribe exercise based on a fixed level of effort 
and compare corresponding workloads to determine whether supplementation might allow for greater workloads and caloric expenditure without a corresponding increase in exertion.

\section{CONCLUSION}

The combination of Rhodiola rosea with a proprietary blend of ingredients, known to improve cellular health and vitality, produced findings that are mixed. This supplement, marketed as NT020 plus Rhodiola rosea, provided no benefit on day-to-day measures of fatigue and energy, but provided notable benefits during and immediately after exercise on these measures. Specifically, the supplementation seemed to make moderately difficult exercise less effortful. Noteworthy is the absence of signs or symptoms suggestive of poor tolerability. This study provides a near optimal framework in terms of design that can be replicated and extended as part of an effort to further evaluate Rhodiola rosea as an isolated compound and/or in combination with other ingredients that make up NT-020 plus Rhodiola rosea.

List of Abbreviations: BMI, Body Mass Index; BP, Blood Pressure; ES, Effect size; HR, Heart rate; RPE, Rate of Perceived Exertion; SD, Standard Deviation.

Author Contributions: M.K., P.B., C.S., and D.W. designed the research. P.L., D.W., and M.K., conducted the research. M.K. ran statistical analysis. M.K, P.L, P.B., and D.W., wrote the manuscript. M.K. had primary responsibility for the content.

Competing Interests: P.B. and C.S. have financial interests in Natura Therapeutics, which is the company that sells the product tested in the submitted study.

Acknowledgements and Funding: We would like to thank P. Bickford and C. Sanberg for their contributions with the study. This work was supported by a grant from the Florida High Tech Corridor.

\section{REFERENCES}

1. Multivitamin/mineral Supplements- National Institutes of Health: Office of Dietary Supplements [https://ods.od.nih.gov/factsheets/MVMS-HealthProfessional/] October 17, 2019

2. What does caffeine do to your body? [http://www.medicalnewstoday.com/ articles/285194.php] October 16, 2017

3. Stear SJ, Castell LM, Burke LM, Spriet LL: JSM reviews: A-Z of nutritional supplements: dietary supplements, sports nutrition foods and ergogenic aids for health and performance Part 6. British Journal of Sports Medicine 2010, 44:297-298.

4. Bucci LR: Selected herbals and human exercise performance. The American Journal of Clinical Nutrition 2000, 72(2 Suppl):624S-36S. 
5. Sellami M, Slimeni O, Pokrywka A, Kuvacic G, Hayes LD, Milic M, Padulo J: Herbal medicine for sports: A review. Journal of the International Society of Sports Nutrition 2018, 15(14):1-14.

6. Ishaque S, Shamseer L, Bukutu C, Vohra S: Rhodiola rosea for physical and mental fatigue: a systemic review. BMC Complementary and Alternative Medicine 2012, 12:70.

7. Brown R, Gerbarg P, Remazanov Z: Rhodiola rosea: A phytomedicinal overview. HerbalGram 2002, 56:40-52.

8. Bickford P, Tan J, Shytle D, Sanberg C, El-Badri N, Sanberg P: Nutraceuticals synergistically promote proliferation of human stem cells. Stem Cells and Development 2006, 15:118-123.

9. Shytle D, Ehrhart J, Tan J, Vila J, Cole M, Sanberg C, Sanberg P, et al.: Oxidative stress of neural, hematopoietic, and stem cells protection by natural compounds. Rejuvenation Research 2007, 10(2):173-8.

10. Choi SY: Anti-fatigue effects of Rhodiola rosea extract. Planta Medica 2016, 82(S 01):S1S381.

11. Torri E, Lemos M, Caliari V, Kassuya CA, Bastos JK, Andrade SF: Anti-inflammatory and antinociceptive properties of blueberry extract (Vaccinium corymbosum). Journal of Pharmacy and Pharmacology 2007, 59(4):591-596.

12. Mancini E, Beglinger C, Drewe J, Zanchi D, Lang UE, Borgwardt S: Green tea effects on cognition, mood and human brain function: A systematic review. Phytomedicine 2017, 34:26-37.

13. Horii Y, Fujisaki Y, Fuyuki R, Nagai K: L-Carnosine's dose-dependent effects on muscle sympathetic nerves and blood flow. Neuroscience Letters 2015, 591:144-148.

14. Chiang CM, Ismaeel A, Griffis RB, Weems S: Effects of Vitamin D Supplementation on Muscle Strength in Athletes. Journal of Strength and Conditioning Research 2017, 31(2):566-574.

15. Small BJ, Rawson KS, Martin C, Eisel SL, Sanberg CD, McEvoy CL, Sanberg PR, et al.: Nutraceutical intervention improves older adults' cognitive functioning. Rejuvenation Research 2014, 17(1):27-32.

16. Acosta S, Jernberg J, Sanberg CD, Sanberg PR, Small BJ, Gemma C, Bickford PC: NT-020, a natural therapeutic approach to optimize spatial memory performance and increase neural progenitor cell proliferation and decrease inflammation in the aged rat. Rejuvenation Research 2010, 13(5):581-8.

17. Flowers A, Lee JY, Acosta S, Hudson C, Small B, Sanberg CD, Bickford PC: NT-020 treatment reduces inflammation and augments $\mathrm{Nrf}-2$ and Wnt signaling in aged rats. Journal of Neuroinflammation 2015, 12(1):174.

18. American College of Sports Medicine: ACSM's guidelines for exercise testing and prescription. Baltimore, MD: Lippincott Williams and Wilkins; 2018.

19. Wasserman K, Hansen JE, Sue DY, Casaburi R, Whipp BJ: Principle of Exercise Testing and Prescription. Philadelphia (PA): Lippincott Williams \& Wilkins; 1999:115-42. 
20. Borg G: Borg's perceived exertion and pain scales. Champaign, IL, US: Human Kinetics; 1998.

21. Curran SL, Andrykowski MA, Studts JL: Short form of the profile of mood states (POMSSF): psychometric information. Psychological Assessment 1995, 7:80-83.

22. Coyle EF, Gonzalez-Alonso J: Cardiovascular drift during prolonged exercise: new perspectives. Exercise and Sport Science Reviews 2001, 29:88-92.

23. Chiang HM, Chen HC, Wu CS, Wu PY, Wen KC: Rhodiola plants: Chemistry and biological activity. Journal of Food and Drug Analysis 2015, 23(3):359-369.

24. Sist P, Tramer F, Lorenzon P, Urbani R, Vrhovsek U, Bernareggi A, Sciancalepore M: Rhodiola rosea, a protective antioxidant for intense physical exercise: An in vitro study. Journal of Functional Foods 2018, 48:27-36.

25. Tsai TW, Chang CC, Liao SF, Liao YH, Hou CW, Tsao JP, Cheng IS: Effect of green tea extract supplementation on glycogen replenishment in human skeletal muscle. British Journal of Nutrition 2017, 117(10):1343-1350.

26. Korf EA, Kubasov IV, Vonsky MS, Novozhilov AV, Runov AL, Kurchakova EV, Matrosova EV, et al.: Green tea extract increases the expression of genes responsible for regulation of calcium balance in rat slow-twitch muscles under conditions of exhausting exercise. Bulletin of Experimental Biology and Medicine 2017, 164(1):6-9. 